\title{
The evolution of the internal rotation of solar-type stars
}

\author{
Maria Pia Di Mauro ${ }^{1}$, Rita Ventura ${ }^{2}$, Daniela Cardini ${ }^{1}$, \\ Jørgen Christensen-Dalsgaard ${ }^{3}$, Wojciech A. Dziembowski ${ }^{4}$ \\ and Lucio Paternò ${ }^{2}$ \\ ${ }^{1}$ INAF-IAPS Istituto di Astrofisica e Planetologia Spaziali, Via del Fosso del Cavaliere 100, \\ 00133 Roma, Italy email: maria.dimauro@inaf.it \\ ${ }^{2}$ INAF-Astrophysical Observatory of Catania, Via S. Sofia 78, 95020, Catania, Italy \\ ${ }^{3}$ Stellar Astrophysics Centre, Department of Physics and Astronomy, Aarhus University, Ny \\ Munkegade 120, DK-8000 Aarhus C, Denmark \\ ${ }^{4}$ Warsaw University Observatory, Al. Ujazdowskie 4, 00-478 Warszawa, Poland
}

\begin{abstract}
We discuss the potential of asteroseismic inversion to study the internal dynamics of solar-type stars and to reconstruct the evolution of the internal rotation from the main sequence to the red-giant phase. In particular, we consider the use of gravity and mixed modes and the application of different inversion methods.
\end{abstract}

Keywords. stars: oscillations, stars: rotation, stars: evolution, Sun: helioseismology, Sun: evolution, Sun: rotation

\section{Introduction}

The knowledge of the variation of the stellar rotation with radius and time is essential for a complete understanding of the evolution of the internal properties of the Sun and other solar-type stars and for explaining the loss of angular momentum occurring from the main sequence to late evolution stages.

In addition, the determination of the internal angular velocity would help to constrain dynamo theories and the connection between rotation and convection and to establish the role played by the mixing in the stellar core.

Until recently, these and similar issues could not be addressed, since the internal rotation was inaccessible to observation. In the 90's, the development of helioseismology and the high quality of oscillation data provided by the satellite $\mathrm{SOHO}$ (Scherrer et al. 1995) and ground-based networks demonstrated that the internal rotation of the Sun could be investigated through the measurement of the splittings of the oscillation frequencies. In fact, the rotation breaks the spherical symmetry of the solar structure and splits the frequency of each oscillation mode by an amount whose magnitude is related to the angular velocity (Cowling \& Newing 1949).

More recently, the unprecedented frequency resolution supplied by the space missions Kepler (Borucki et al. 2010) and CoRoT (Baglin et al. 2006) has allowed accurate measurements of rotational splittings in stars in different evolutionary stages, and in particular in red-giant phase (Beck et al. 2012, Mosser et al. 2012, Deheuvels et al. 2012). The results indicate a fast core rotation in stars ascending the red-giant branch and a spin-down of the core in the red-clump stars. This implies that a significant angular momentum transport takes place during the last stages of red-giant branch evolution. 
In this article we consider the use of inversion techniques, applied with success to the case of the Sun, in order to reconstruct rotation history in solar-type stars from the main sequence to the red-giant phase of evolution.

\section{Asteroseismic inversion}

Asteroseismic inversion is a powerful tool that allows one to estimate the physical properties of the stars, by solving integral equations expressed in terms of the experimental data.

Inversion techniques are well known and applied with success to several branches of physics. Applications to helioseismic data have been studied extensively and inversion methods and techniques have been reviewed and compared by several authors, leading to extraordinary results about the internal rotation of the Sun (e.g., Di Mauro 2003).

In order to quantify the internal rotation it is possible to invert the following equation, relating the set of the observed splittings $\delta \nu_{n, l}$ to the internal rotation $\Omega(r)$ :

$$
\delta \nu_{n, l}=\int_{0}^{R} \mathcal{K}_{n, l}(r) \frac{\Omega(r)}{2 \pi} d r+\sigma_{n, l}
$$

where $\sigma_{n, l}$ are the errors in the measured splittings, and $\mathcal{K}_{n, l}(r)$ are the kernel functions, calculated on the stellar model for each mode $i \equiv(n, l)$. The inverse problem consists of finding the function $\Omega(r)$ by using a finite set of observed splittings $i=1, \ldots, M$ and their associated errors $\sigma_{i}$. This is an 'ill-posed' problem which can be solved by employing an appropriate technique.

The present article deals with the application of the Optimally Localized Averaging (OLA) method based on the original idea of Backus \& Gilbert (1970), which allows one to estimate a localized weighted average of the angular velocity $\bar{\Omega}\left(r_{0}\right)$ at selected target radii ' $\left\{r_{0}\right\}$ ' by means of a linear combination of all the data:

$$
\bar{\Omega}\left(r_{0}\right)=\sum_{i}^{M} c_{i}\left(r_{0}\right) 2 \pi \delta \nu_{i}=\sum_{i=1}^{M} c_{i}\left(r_{0}\right) \int_{0}^{R} \mathcal{K}_{i}(r) \Omega(r) d r
$$

where $c_{i}\left(r_{0}\right)$ are the inversion coefficients and $K\left(r_{0}, r\right)=\sum_{i=1}^{M} c_{i}\left(r_{0}\right) \mathcal{K}_{i}(r)$ are the socalled averaging kernels. We look for the coefficients $c_{i}\left(r_{0}\right)$ which minimize the propagation of the errors and the spread of the averaging kernels.

The errors of the solutions are given by $\delta \bar{\Omega}\left(r_{0}\right)=\left[\sum_{i=1}^{M} c_{i}^{2}\left(r_{0}\right) \sigma_{i}^{2}\right]^{1 / 2}$, while the radial spatial resolution is assumed to be the half-width at half-maximum of the averaging kernels.

The code, developed for the solar rotation (Paternò et al. 1996), has been adapted here to be applied to any evolution phase. We also considered the method in the variant form proposed by Pijpers \& Thompson (1992) and known as SOLA (Subtractive Optimally Localized Averaging), making attempts to fit the averaging kernel to a Gaussian function of appropriate width, centred at the target radius (Di Mauro \& Dziembowski 1998).

\section{Inversion results}

The properties of the inversion depend both on the mode selection and on the observational errors, which characterize the mode set to be inverted. The large set of data available for the Sun, including modes with harmonic degree $l=1-100$, allows to sound the rotational profile from the core to the upper layers. However, inferences of the interior 

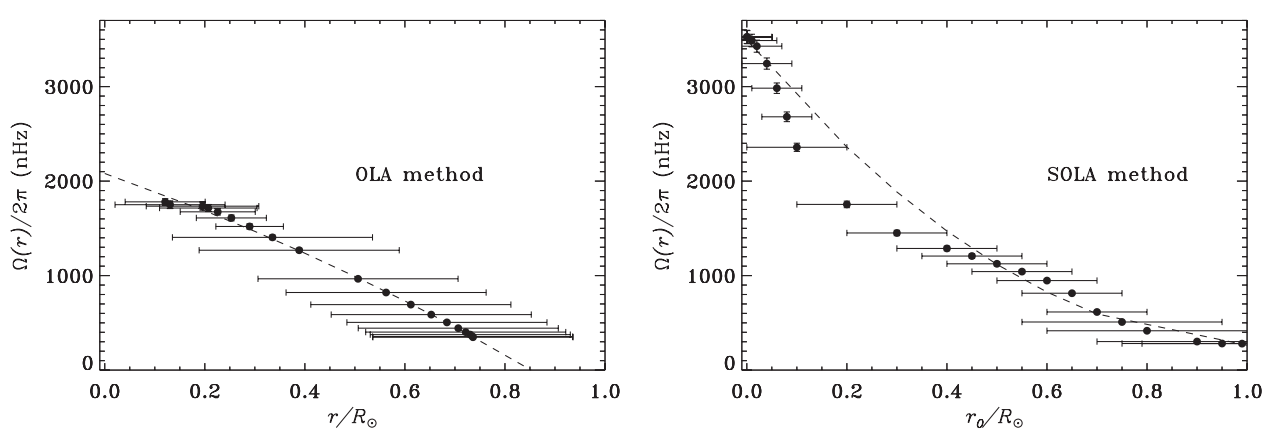

Figure 1. Internal rotation of the Sun as deduced by inversion of $l=1$ rotational splittings observed with GOLF/SOHO with the OLA (left panel) and the SOLA (right panel) methods. The radial resolution is equal to the width of the averaging kernels, while the error in the solution is the standard deviation calculated on the observational uncertainties of the data. Dashed lines are polynomial fitting functions. Data have been kindly provided by the GOLF/SOHO team.
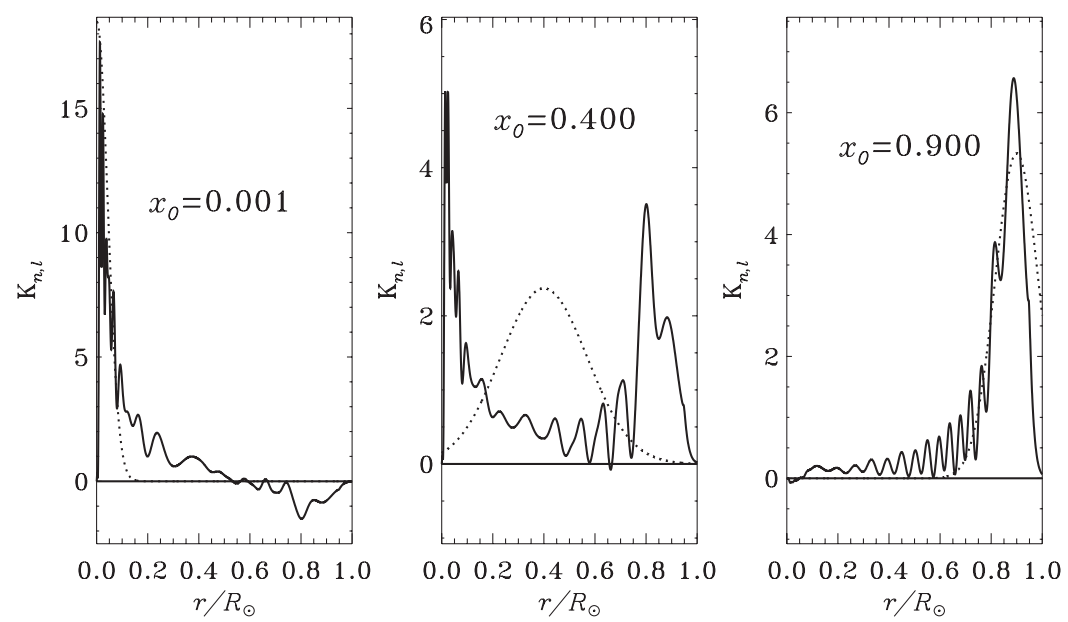

Figure 2. Averaging kernels (solid lines) at selected target radii for the inversion of $l=1$ GOLF/SOHO data as obtained by using the SOLA method and including g modes. Dashed lines indicate the Gaussian target functions.

of stars other than the Sun appear to be much more complicated and less outstanding in terms of achievable results. The large stellar distances, the point-source character of the stars, and the low amplitude of the oscillations restrict the asteroseismic studies to the use of small sets of data characterized by modes with only low harmonic degrees $(l \leqslant 3)$.

At present, rotational splittings are not yet available for main-sequence stars other than the Sun. However, it is possible to treat the Sun as a star by inverting a set of only low-degree modes. The variation of the Sun's angular velocity with the radius, shown in Fig. 1, has been determined by inversions of a set of 15 rotational splittings with $l=1$, including $6 \mathrm{~g}$ modes and $9 \mathrm{p}$ modes, detected by the GOLF instrument flying on $\mathrm{SOHO}$ (García et al. 2007, 2008). The results in the core and below the surface agree with those of García et al. (2011) obtained by inverting a full set of data with $l=1-100$ : the core appears to rotate faster than the surface. The ratio $\Omega_{\mathrm{c}} / \Omega_{\mathrm{surf}} \simeq 4-8$, where $\Omega_{\mathrm{c}}$ and $\Omega_{\text {surf }}$ are respectively the angular velocity in the core and at the surface. It is possible to notice that the SOLA method allows one to find solutions better localized than the OLA method, but both fail to localize solutions between $0.2-0.8 R_{\odot}$ as shown by the averaging kernels plotted in Fig. 2. 
We have then considered the star KIC 4448777 located at the beginning of the ascending red-giant branch observed by the Kepler satellite (Di Mauro et al. 2013). The present asteroseismic inversion has been carried out by employing the rotational splittings of 15 mixed modes with $l=1$. The gravity component of the mixed modes allows one to find solutions well localized in the core, while the acoustic component of the modes give the possibility to localize solutions in the upper layers. As in the case of the Sun, it is not possible to obtain localized kernels between the core and the upper layers.

We confirm the previous findings in other red giants (Deheuvels et al. 2012) that the core rotates faster than the surface, and in the considered star, at a rate of about $955 \pm 25 \mathrm{nHz}$. The angular velocity, through the largest part of the convection zone, from the core towards the surface, decreases with increasing distance from the centre, reaching a value of about $84 \pm 7 \mathrm{nHz}$ at $0.9 R$, so that $\Omega_{\mathrm{c}} / \Omega_{\text {surf }} \simeq 10-12$. The spectroscopic value of $v \sin i$ is not known.

\section{Conclusions}

It is possible to conclude that helioseismic tools can be extended to other stars and the internal rotation of solar-type stars can be inferred by using inversion techniques to reconstruct the evolution of stellar rotation. Mixed modes, like the gravity modes, allow one to find solutions in the inner core.

The results seem to indicate that from the main sequence to early red-giant phases the rotation slows down both in the envelope and in the core, while the ratio $\Omega_{\mathrm{c}} / \Omega_{\mathrm{surf}}$ is almost conserved.

Considering the fact that the internal rotation of the core, as predicted by the current theoretical models of red giants, is higher compared to our results, it is necessary to investigate more efficient mechanisms of angular momentum transport acting on the appropriate timescales during the different phases of stellar evolution.

Finally, we expect that measurements of rotational splittings, which will be soon available for subgiants and more evolved red-giant stars, will shed some light on the above picture and on the question of angular momentum transport.

\section{References}

Backus, G. E. \& Gilbert, F. 1970, Phil. Trans. R. Soc. London, 266, 123

Baglin, A., Auvergne, M., Boisnard, L., et al. 2006, in: 36th COSPAR Scientific Assembly Plenary Meeting, 36, 3749

Beck, P., Montalbán, J., Kallinger, T., et al. 2012, Nature, 481, 55

Borucki, W. J., Koch, D., Basri, G., et al. 2010, Science, 327, 977

Cowling, T. G. \& Newing, R. A. 1949, ApJ, 109, 149

Deheuvels, S., García, R. A., Chaplin, W. J., et al. 2012, ApJ, 756, 19

Di Mauro, M. P. 2003, Lectures Notes in Physics, 599, 31

Di Mauro, M. P. \& Dziembowski, W. A. 1998, MemSAIt, 69, 559

Di Mauro, M. P., Cardini, D., Ventura, R., et al. 2013, in: 40th Liège International Astrophysical Colloquium Ageing Low Mass Stars: From Red Giants to White Dwarfs, EPJ Web of Conferences, 43, 03012

García, R. A., Turck-Chièze, S., Jiménez-Reyes, S. J., et al. 2007, Science, 316, 1591

García, R. A., Mathur, S., Ballot, J., Eff-Darwich, A., Jiménez-Reyes, S. J., \& Korzennik, S. G. 2008, Solar Phys., 251, 119

García, R. A., Salabert, D., Ballot, J., et al. 2011, JPhCS, 271, 2046

Mosser, B., Goupil, M.-J., Belkacem, K., et al. 2012, A\&A, 548, A10

Paternò, L., Sofia, S., \& Di Mauro, M. P. 1996, A\&A, 314, 940

Pijpers, F. P. \& Thompson, M. J. 1992, A\&A, 262, L33

Scherrer, P. H., Bogart, R. S., Bush, R. I., et al. 1995, Solar Phys. 162, 129 